\title{
Neue genetische Marker als Prognosefaktoren beim Mammakarzinom
}

Die erheblichen Unterschiede in der Prognose von Brustkrebspatientinnen beruhen zum Teil auf genetischen Variationen. Erkenntnisse über den Keimbahn-Genotyp können zusätzlich zu bekannten Prognosefaktoren Informationen zum Krankheitsverlauf liefern.

\begin{abstract}
7 um Zusammenhang zwischen Keimbahnmutationen und dem brustkrebsspezifischen Überleben gibt es eine Reihe von Studien. Diese detektierten eine Vielzahl von Einzelnukleotid-Polymorphismen (SNPs) mit möglicher Relevanz für den Krankheitsverlauf. Auch genomweite Assoziationsstudien (GWAS) identifizierten Suszeptibilitäts-Allele für eine Reihe von normalen und Krankheits-Phänotypen. Einen Zusammenhang mit dem Überleben beim Mammakarzinom vermochten beide Wege nicht herzustellen. Im Bestreben, mit dem brustkrebsspezi-
\end{abstract}

fischen Überleben assoziierte genetische Marker zu identifizieren, setzten Qi Guo und Kollegen eine große Metaanalyse von Studien mit insgesamt 37.954 Brustkrebspatientinnen europäischer Abstammung auf, darunter 2.900 karzinombedingte Todesfälle. Jede Studie war genotypisiert für 200.000-900.000 SNPs mittels verschiedener GenotypisierungsArrays. Über ein Referenz-Panel des 1.000-Genome-Projekts imputierten die Wissenschaftler die Genotypen für 9 Millionen Varianten. In die Auswertung bezogen sie auch subtypspezifische Analysen mit ER(Östrogenrezeptor)-positi- ven und ER-negativen Patientinnen ein. Im Ergebnis wies eine einzelne imputierte SNP namens „rs2059614“ auf Chromosom 11 eine statistisch signifikante Assoziation mit der brustkrebsspezifischen Mortalität bei ER-negativen Patientinnen auf und ein zweiter Locus (rs2059614) auf der genomweiten Ebene.

Fazit: Diese Studie zur Assoziation genetischer Variationen mit dem brustkrebsspezifischen Überleben ist bisher die größte ihrer Art. Die Ergebnisse bestätigen die prognostische Bedeutung des Keimbahn-Genotyps zusätzlich zu den bisherigen prädiktiven Faktoren.

Wolfgang Zimmermann

Guo Q et al. Identification of novel genetic markers of breast cancer survival. J Natl Cancer Inst. 2015 Apr 18;107(5). pii: djv081.

\section{Brusterhaltende Operation: Brachytherapie für jüngere Patientinnen?}

\section{Die Bedeutung einer Teilbestrahlung der weiblichen Brust nach Lumpektomie bei jüngeren Patientinnen mit einem Mammakarzinom wird kontrovers diskutiert. Verlässliche Selektionskriterien könnten die Therapieentscheidung erleichtern. Dem gingen Wissenschafler am MD Anderson Cancer Center in Houston, TX/USA, nach.}

$\mathrm{n}$ den letzten Jahren erhielten ältere Brustkrebspatientinnen nach Lumpektomie zunehmend eine interstitielle Brachytherapie anstelle der Ganzbrustbestrahlung im Rahmen eines adjuvanten radiologischen Therapiekonzepts. In den USA bestehen jedoch erhebliche Differenzen in den Empfehlungen seitens der Fachgesellschaften zur Teilbrustbestrahlung jüngerer Patientinnen nach brusterhaltender Operation. Keine Leitlinie zieht den ÖstrogenrezeptorStatus (ER-Status) als alleiniges Ausschlusskriterium für eine Brachytherapie heran.

Die aktuell unzureichende Datenlage vermag die differierenden Empfehlungen nicht zu versöhnen. Während Einigkeit über jüngeres Alter und ER-Negativität als Risikofaktoren für erhöhtes Rezidivrisiko nach Lumpektomie herrscht, bleibt unklar, ob eine Brachytherapie weniger protektiv wirkt als die Ganzbrustbestrahlung. Es besteht daher die Notwendigkeit zur Aufstellung von Selektionskriterien für die Teilbrustbestrahlung jüngerer Patientinnen.

In einer nationalen Kohortenstudie mit den Daten von 45.884 Brustkrebspatientinnen im Alter zwischen 18 und 64 Jahren, die im Zeitraum von 2003 bis 2010 lumpektomiert wurden mit nachfolgender adjuvanter Therapie, stellte die Arbeitsgruppe um Grace L. Smith einen direkten Vergleich zwischen Brachytherapie und Ganzbrustbestrahlung an. Die Forscher berücksichtigten dabei: _das Therapiemuster,

_das Risiko einer späteren Mastektomie, _die Behandlungskosten.

Im Beobachtungszeitraum erhöhte sich der Anteil der adjuvant teilbrustbe- strahlten Patientinnen unter 50 Jahren von 0,6 auf $4,9 \%$, in der Gruppe der über 50 -Jährigen von 2,2 auf $11,3 \%$. Das Alter bestimmte in höherem Maß die Therapiewahl als der Östrogenrezeptorstatus: $17 \%$ der jüngeren Patientinnen erhielten eine Brachytherapie, $32 \%$ eine Ganzbrustbestrahlung.

Das entsprechende Verhältnis ER-positiver gegenüber ER-negativer Patientinnen betrug $41 \mathrm{zu} 44 \%$. ER-negative Patientinnen unter 50 Jahren wiesen das höchste absolute Risiko für eine posttherapeutische Mastektomie auf: $24 \%$ nach Brachytherapie und nur 9\% nach Ganzbrustbestrahlung.

Fazit: Der Östrogenrezeptorstatus eignet sich als wichtiger diskriminatorischer Faktor zur Einschätzung des Risikos für eine Mastektomie nach vorhergehender Brachytherapie bzw. Ganzbrustbestrahlung bei jüngeren Frauen mit Brustkrebs.

Wolfgang Zimmermann

Smith GL et al. Utilization and Outcomes of Breast Brachytherapy in Younger Women. Int J Radiat Oncol Biol Phys. 2015;93(1):91-101. 